\title{
Research of the Low-voltage Warning Based on Statistical Inference
}

\author{
Sheng Liu ${ }^{a}$ \\ State Grid Fujian Electric Power Company Limited, Fuzhou 350000, China. \\ aliu_sheng@fj.sgcc.com.cn
}

Keywords: low-voltage discrimination; warning; urgent index.

\begin{abstract}
In this paper, we refer the discriminate model of low-voltage and urgencies index as an important research content. Formulate the distribution function and calculate the probability of occurrence of each interval by analyzing the data distribution of the low-voltage. Urgencies indicators are set based on the probability, to mention reference for the staff of the grid and improve the work efficiency of the power grid. In addition, the paper also studies the voltage fluctuations, and designs the formula of voltage fluctuations rate, which severs as a parameter to calculate the urgency index, to enhance the actual role of the urgencies index. The empirical results show the effectiveness and high precision of the discriminate model and we can promote the use of it.
\end{abstract}

\section{Introduction}

During the operation of power grid, low-voltage is a common situation. The results caused by low-voltage can be serious. At present, low-voltage mainly happens in rural distribution network, leading to the results that users can't use electricity normally, high power electrical applications cannot be used, and power grid corporations receive complains from users. Furthermore, other abnormal situations such as the three-phase unbalance, low power factor, which caused by low voltage, affect the normal operation of the grid.

Concerning the hazards caused by low-voltage towards power systems and users, many experts have done lots of researches. Li Caixia, Zhao Yuzhen and Guo Jianfeng (2007) analyze the reasons for the low voltage and the damages to electrical equipment caused by it. They put forward a variety of measures to prevent low voltage operation as well. Ma Xiang (2010) addressed the hazards to electrical equipment and power grid caused by the low-voltage specifically and then put forward some preventive measures. In a word, transformation on the low voltage is imminent.

In order to transform the low-voltage power grid, the first step is to identify where the low voltage happens. But for now, the researches on this are limit. Weng Xi'an (2009) analyzes the influences between differences equipment and measurements on the loss of low-voltage power grid based on the transformer and line voltage loss formula, and then propose the corresponding countermeasures. Jin Zhe, Li Jun and Cui Zheng (2010) build Low-voltage geographic information system and area load management and monitoring platform to get the access to the comprehensive real-time data of the low-voltage area load. They propose measures to transform the low-voltage. Li Jun, Li Gang and Sun $\mathrm{Pu}$ (2012) do research on the on-line monitoring and management based on the low-voltage GIS, which combine the low-voltage load area monitoring system and the successfully used low-voltage GIS closely, making the on-line monitoring and analyzing on low-voltage load area possible. At present, the most popular low voltage distribution monitoring is based on the general definition of low voltage. But some serious low-voltage load area cannot be identified, which increase the complication of the smart grid.

Because the current low voltage discrimination rules have its limitation, this article focus on the model of low-voltage discrimination and urgent index evaluation. Through analyzing the distribution of operation low voltage data, fitting the distribution function, and calculating the probability of every interval, we can rate the urgent index of its transformation and make it as a reference for the power grid staff. In this way, the efficiency of the power grid is improved. The empirical results show that the low-voltage monitoring measure proposed in this article has a good effect and high precision, which can provide a reliable basis for power grid renovation work and can be applied widely. 


\section{Modeling}

\subsection{The Definition of Low Voltage}

In the general definition of grid, low voltage is, in a statistical cycle, in the twelve continuous acquisition the output voltage is less than ninety percent of the rated voltage (which means 198V in three-phase four-wire, $90 \mathrm{~V}$ in three-phase three-wire), and more than $180 \mathrm{~V}$ in three-phase four-wire, $80 \mathrm{~V}$ in three-phase three-wire. Serious low voltage is defined as in the twelve continuous acquisitions the output voltage is less than eighty percent of the rated voltage.

From the definition, low voltage can be classified as two kinds: the general low voltage and the severe low voltage. But the two classes can't meet the needs to describe low voltage, so we generate the definition to a continuous abnormal series.

\subsection{Calculation of Voltage Fluctuation Rate}

There isn't a unified method on voltage fluctuation. Usually depend on power grid staff to observe its scatter and judge its fluctuation subjectively, which lack the accuracy and efficiency. So, we propose the voltage fluctuation discrimination method based on fluctuation curve.

Suppose:

$$
\begin{gathered}
V_{1}=\left(v_{1,1}, v_{1,2}, \cdots \cdots, v_{1, n}\right) \\
V_{2}=\left(v_{2,1}, v_{2,2}, \cdots \cdots, v_{2, n}\right) \\
\vdots \\
V_{N}=\left(v_{N, 1}, v_{N, 2}, \cdots \cdots, v_{N, n}\right)
\end{gathered}
$$

It is a record on a load area in $\mathrm{N}$ days, where there are $\mathrm{n}$ records in one day.

Define:

$$
S_{P}=\left(s_{1}, s_{2}, \cdots \cdots, s_{n-2}, s_{n-1}\right)
$$

where $s_{k}=\frac{v_{p, k}-v_{p, k+1}}{V_{0}}, V_{0}$ is the rate voltage in the load area, $p \in[1, N], k \in[1, n-1]$.

Define:

$$
S_{P, \sigma}=\frac{1}{n-1} \sqrt{\sum_{j=1}^{n-1} s_{j}^{2}}
$$

$S_{P, \sigma} \in[0,1]$ which describe the fluctuation in Day P. The bigger it is, the more urgent the transformation is.

\subsection{Low-voltage Urgent Index}

Define the threshold as $V_{a}=0.9 V_{0}, V_{b}=0.82 V_{0}$. Among all the researches, when $V_{b}<V_{p, i}<V_{a}$, it can be defined as general low voltage; when $v_{p, i}<V_{b}$, it can be defined as severe low voltage.

Among all the $\mathrm{n}$ records in a day, the more records in low voltage area, the more urgent the transformation is. In this paper, Logistic Model is used to describe this relationship. Define the impact factor of transformation urgent index as:

$$
\kappa\left(j_{1}\right)=\frac{1}{1+e^{\alpha_{1} j_{1}+\alpha_{2}}}
$$

where $j_{1}=$ num. $\left[w_{1}\right]$ is the number of the elements in $w_{1} . w_{1}$ is a subset of $[1, n]$, which is the set of the subscripts of the voltage which is belongs to $\left[v_{b}, v_{a}\right]$ (such as $v_{p, q} \in\left[v_{b}, v_{a}\right]$, then $q \in w_{1}$ ). Based on the prior information, we can define $j_{1}=12, \kappa\left(j_{1}\right)=0.2, j_{1}=24, \kappa\left(j_{1}\right)=0.7$. Then we can calculate the parameter:

$$
\kappa\left(j_{1}\right)=\frac{1}{1+e^{-0.223 j_{1}+4.067}}
$$


Define:

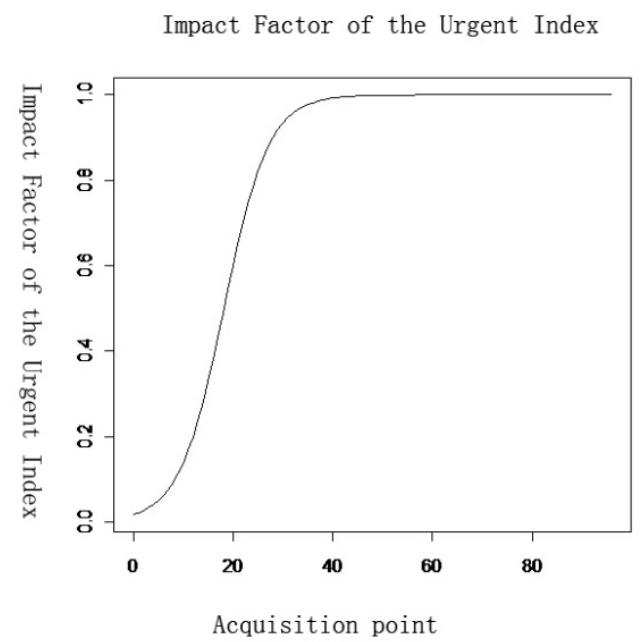

Fig 1. Impact Factor of the Urgent Index Curve

$$
\Pi_{p}=\kappa\left(j_{1}\right)\left(\frac{2}{5 j_{1}} \sum_{i \in \omega_{1}}\left(\frac{v_{p, i}}{v_{b}-v_{a}}-\frac{v_{a}}{v_{b}-v_{a}}\right)+\frac{3}{5}\right)+\sqrt{\frac{a j_{2}}{n}}
$$

where $j_{2}=$ num. $\left[w_{2}\right]$ is the number of the elements in $w_{2} . w_{2}$ is a subset of $[1, n]$, which is the set of the subscripts of the voltage which is lower than $v_{b}$ (such as $v_{p, q}<v_{b}$, then $q \in w_{2}$ ).

During the power delivery process, it is normal that voltage has small fluctuations, which won't influence the stability of the voltage. Define $I_{X}=\left\{\begin{array}{ll}1 & \text { if } X \\ 0 & \text { else }\end{array}\right.$ as an indicator function, $\hat{S}_{P, \sigma}= \begin{cases}1 & \text { if } j_{2} \geq\left[\frac{n}{a}\right] . \text { From the former statement, we have the low-voltage urgent index: } \\ S_{P, \sigma} \text { else } & \end{cases}$

$$
\zeta_{p}= \begin{cases}\Pi_{p} I_{j_{2}<\left[\frac{n}{a}\right]}+I_{j_{2} \geq\left[\frac{n}{a}\right]} & \text { if } S_{p, \sigma}<b \\ \mu_{1}\left(\Pi_{p} I_{j_{2}<\left[\frac{n}{a}\right]}+I_{j_{2} \geq\left[\frac{n}{a}\right]}\right)+\mu_{2} \hat{S}_{P, \sigma} & \text { if } S_{p, \sigma} \geq b\end{cases}
$$

Where $n$ is the number of the elements of voltage acquisition in one day, $a, b, \mu_{1}, \mu_{2}$ are adjustable parameters, $a \in[1, n], b \in(0,1], \mu_{1}, \mu_{2} \in[0,1], \mu_{1}+\mu_{2}=1,\left[\frac{n}{a}\right]$ is the integer part of $\frac{n}{a}$.

\section{Empirical Analysis}

In this paper there are ten load areas selected to be analyze their low-voltage urgency. Discrimination algorithm proposed in this paper and system algorithm used in their own province are used separately to identify the three-phase unbalance. To make the calculation simpler $a=4, b=0.5, \mu_{1}=0.6, \mu_{2}=0.4$. At first, observe the voltage fluctuation in one load area, where the rated power is $400 \mathrm{kv}$, the fluctuation shows as follow: 


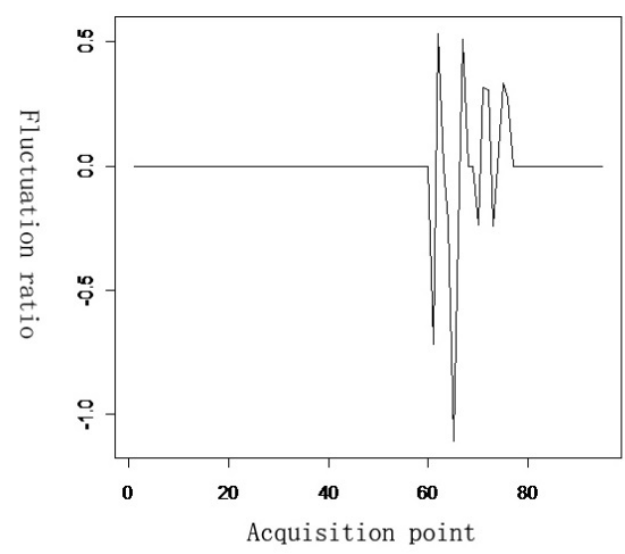

Fig 2. Voltage Fluctuation Curve

From Fig. 2, in this load area most is in the steady state, while there is strong shock in some period.

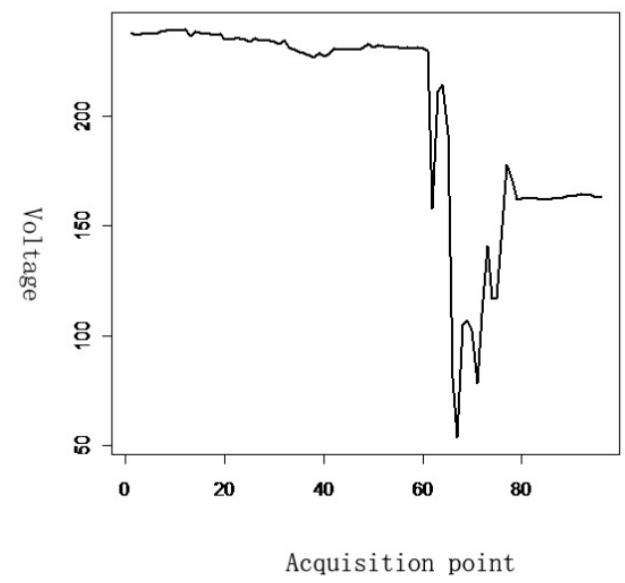

Fig 3. Voltage Change Curve

In general, there is severe low voltage state in some time in this load area, which effects the normal electricity use. Using the algorithm proposed in this paper, the low voltage urgent index is 1 .

Using the same algorithm to analyze other load areas' low voltage situations and comparing with the current algorithm, we can get Table 1.

Table 1. Low Voltage Urgent Index Analysis Table

\begin{tabular}{cccc}
\hline $\begin{array}{c}\text { Lood Area } \\
\text { No. }\end{array}$ & Urgent Index & Based on the current algorithm & $\begin{array}{c}\text { Based on this paper's } \\
\text { algorithm }\end{array}$ \\
\hline 1 & 0.7323 & Low voltage & Low voltage,consider transformation \\
2 & 0.6457 & Normal & Low voltage,consider transformation \\
3 & 0.9443 & Severe Low Voltage & Severe,In urgent need of transformation \\
4 & 0.3215 & Normal & Normal \\
5 & 1.0000 & Normal & Most severe,In urgent need of transformation \\
6 & 0.7526 & Low voltage & Low voltage,consider transformation \\
7 & 0.8477 & Low voltage & Severe,In urgent need of transformation \\
8 & 1.0000 & Severe Low Voltage & Most severe,In urgent need of transformation \\
9 & 0.8227 & Low voltage & Severe,Need to transform \\
10 & 0.5933 & Normal & Low voltage,consider transformation \\
\hline
\end{tabular}


Using the current algorithm, the low voltage and the severe low voltage can be distinguished, while the monitoring is not accurate. Through empirical analysis, we can calculate the low voltage urgent index to improve the efficiency of power grid and to guarantee the safety use of electricity.

\section{Conclusion}

Monitoring and Warning on low voltage is an important task in power grid, for it influences the electricity use for every user. In this paper a model is established based on statistic theory, which analyzes that low voltage changing deeply, determines the working conditions of low voltage accurately and quantifies the low voltage transformation urgency index. This model can offer power grid staff a useful tool to manage low voltage, improve their work efficiency and increase the power grid economic benefit and social benefit. With the empirical analysis, the discrimination proposed in this paper has good effect and high precision, which can be widely used.

\section{References}

[1]. Jin Zhe, Li Jun, Cui Zheng. "Low Voltage" Monitoring System Previous Studies and Initial Applications[J]. Rural Electrification. (2011) No.4, p.5-7.

[2]. Li Caixia, Zhao Yuzhen, Guo Jianfeng. Harm and Preventive Measures of Low Voltage Movement in Electrical Network. Coal Technology. Vol.26 (2007) No.1, p.37-39.

[3]. Li Jun, Li Gang, Sun Pu. "Low Voltage" on-line monitoring and analysis management[J]. Rural Electrification. (2012) supplement, p.42-44.

[4]. Ma Xiang. Detriment of Power Grid Low Voltage Operation to Electric Equipment and Power System and Preventive Measures[J]. Value Engineering. Vol.29 (2010) No.27, p.135-136.

[5]. Weng Xi'an. The Analysis on Village Low-Pressure Electrical Distribution Network. Popular Utilization of Electricity. (2009) No.3, p.36-38. 